\title{
Dimensões Pessoais e Contextuais da Excelência: Estudo com Cientistas Brasileiros
}

\author{
Maria Célia Bruno Mundim¹, Solange Muglia Wechsler \\ Pontifícia Universidade Católica de Campinas, Campinas-SP, Brasil \\ Leandro da Silva Almeida \\ Universidade do Minho, Portugal
}

\section{RESUMO}

Este estudo teve como objetivo investigar as variáveis pessoais e contextuais que influenciam a produção da excelência em pesquisadores brasileiros. Participaram do estudo 109 pesquisadores, bolsistas de produtividade do CNPq nível 1, selecionados pela plataforma Lattes. O Questionário do Perfil de Excelência foi administrado on-line e composto de perguntas semiabertas sobre aspectos cognitivos, de personalidade, biográficos e contextuais. A análise das respostas por área científica e gênero foram feitas pelo qui-quadrado. Houve diferenças significativas entre homens e mulheres das diferentes áreas científicas quanto à fase de interesse pela profissão, na idade adulta para mulheres e infância para homens, bem como em relação às características persistência e inconformismo, mais valorizadas pelas mulheres para a carreira científica. Os resultados sugerem que o gênero e a área de conhecimento influenciam mais as variáveis pessoais do que as contextuais na excelência científica.

Palavras-chave: excelência; produtividade; pesquisador; personalidade; gênero.

\section{ABSTRACT - Personal and Contextual Dimensions of Excellence: Study with Brazilian Scientists}

This study aimed to investigate personal and contextual variables that influence the production of excellence in Brazilian researchers. For this, 109 researchers were selected from the Lattes Platform, classified as level 1 by the CNPQ agency. The Excellence Profile Questionnaire was administered online and comprised of semi-open questions about cognitive, personality, biographical and contextual aspects. The analysis of the answers by scientific area and gender was made using the chi-square test. Significant differences were found between men and women from the different scientific areas regarding the career interest phase, in adulthood for women and childhood for men. Gender differences were related to the characteristics of persistence and nonconformity, which was more valued by women for a scientific career. The results suggest that gender and the area of knowledge influence personal variables more than contextual variables in scientific excellence.

Keywords: excellence; personality traits; contextual factors; academic researchers.

\section{RESUMEN - Dimensiones Personales y Contextuales de la Excelência: Estudio com Científicos Brasileños}

Este estudio tuvo como objetivo investigar variables personales y contextuales que influencian la producción de la excelencia en investigadores brasileños. Participaron del estudio 109 investigadores, becarios de productividad del CNPq nivel 1, seleccionados por plataforma Lattes. El Cuestionario del Perfil de Excelencia fue administrado on-line y compuesto de preguntas semi abiertas sobre aspectos cognitivos, de personalidad, biográficos y contextuales. El análisis de las respuestas por área científica y género fueron hechas por el Chi-cuadrado. Se observaron diferencias significativas entre hombres y mujeres de las diferentes áreas científicas con respecto a la fase de interés por la profesión, en la edad adulta para las mujeres y en la infancia para los hombres, así como en relación con las características persistencia e inconformismo más valoradas por las mujeres para la carrera científica. Los resultados sugieren que, con respecto a la excelencia científica, el género y el área de conocimiento influyen más en las variables personales que en las contextuales.

Palabras clave: excelencia; rasgos de la personalidad; factores contextuales; investigadores académicos.

A excelência é um construto recente e, portanto, pouco conhecido na ciência psicológica. Esse conceito surgiu nos últimos anos associada ao desenvolvimento da Psicologia Positiva, estando mais centrado nas características positivas do indivíduo, tais como o talento, a sabedoria, a criatividade, a autenticidade, a liderança e a perseverança (Hutz \& Pacico, 2016; Seligman \& Csikszentmihalyi, 2000).

Por excelência entende-se a expertise, a capacidade ou o desempenho superior de um indivíduo frente aos pares em uma mesma área de atuação (Shavinina, 2009), aparecendo por vezes referenciada na literatura 
como sinônimo de talento e superdotação (Araújo, Cruz, \& Almeida, 2007), criatividade (Olszewski-Kubilius \& Thomson, 2015) ou sabedoria (Monteiro, Almeida, \& Vasconcelos, 2009). Cabe, entretanto, identificar os determinantes da excelência, em vista da sua raridade e de seu forte impacto ao êxito profissional dos indivíduos (Garcia-Santos, Almeida, \& Werlang, 2012).

De acordo com Trost (2000), a excelência resulta da interação das características pessoais (traços de personalidade, habilidades cognitivas e aspectos motivacionais) com os fatores ambientais (família, escola, dentre outros). Semelhantemente, Almeida e Wechsler (2015) referem que a excelência profissional ocorre por meio da confluência das características psicológicas com a motivação, a cognição e a personalidade. Já mais na linha da superdotação, Renzulli (2005) explica o talento superior por meio da interação da criatividade com as habilidades acima da média e com o comprometimento com a tarefa. O seu modelo, conhecido como Teoria dos Três Anéis, tanto explica a superdotação em geral expressa desde a infância como o talento em determinados campos de atuação.

\section{Variáveis influenciáveis na excelência}

No que se refere ao talento científico precoce, Dou, Hazari, Dabney, Sonnert, \& Sadler (2019) verificaram que certas experiências na infância, principalmente na interação com amigos ou familiares sobre ciências, podem contribuir favoravelmente para o interesse nessa área. Dabney, Chakraverty e Tai (2013) também notaram a influência familiar sobre a curiosidade inicial dos estudantes para as ciências. Portanto, a família tem grande importância no desenvolvimento do talento de um indivíduo, apesar de haver pais que discriminem a educação entre os filhos de acordo com o estereótipo de gênero, favorecendo o envolvimento do menino em atividades escolares e o envolvimento da menina em atividades domésticas (Buchmann, DiPrete, \& McDaniel, 2008).

Além do apoio dos pais, a escola e a qualidade do professor têm contribuições relevantes para o desenvolvimento do talento em ciências (Osborne, Erduran, \& Simon, 2004; Pruitt \& Wallace, 2012). Aos professores de ciências, por exemplo, é atribuído o estímulo das habilidades e do pensamento criativo dos seus alunos (Bakir \& Öztekin, 2014) desde a educação primária (Baron \& Chen, 2012) até o ensino superior (Russell, Hancock, \& McCullough, 2007). Nesse sentido, a mentoria por parte dos professores torna-se um elemento importante para direcionar a aprendizagem e encorajar atitudes frente aos conhecimentos científicos (Fleming, Burnham, \& Huskins, 2012; Gonzalez, 2001).

Ao analisar as experiências pregressas e as memórias de cientistas a respeito das pessoas significativas que influenciaram suas carreiras, foram observadas como importantes as orientações formais e informais de pais e professores (Jones, Taylor, \& Forrester, 2011). Outros fatores percebidos como decisivos para a escolha de meninos e meninas pela atividade científica foram: a) a identificação com determinada disciplina vista na escola, b) o modelo do professor e c) o envolvimento dos pais na promoção do sucesso acadêmico do filho (Salehjee \& Watts, 2015).

Ainda quanto à identificação e ao desenvolvimento do talento para as ciências, Renzulli, Siegle, Reis, Gavin e Reed (2009) notaram as seguintes características pessoais dos estudantes que se interessaram pela área: (a) curiosidade sobre o modo como as coisas são feitas e os processos científicos, (b) pensamento criativo sobre problemas, (c) entusiasmo na discussão de temas científicos, e (d) interesse nos tempos livres pela leitura de questões relacionadas às ciências. Gould e Weeks (2005) também observaram em jovens talentosos grande facilidade para compreender conceitos científicos, assim como uma grande motivação intrínseca pela sua aprendizagem sugerindo que, para eles, aprender ciências é uma diversão.

Embora haja uma tendência pelo surgimento de interesse pelas ciências na pré-adolescência e adolescência (Tai, Qi Liu, Maltese, \& Fan, 2006), a aspiração para esse campo de atuação está, dentre outros fatores, fortemente relacionada ao gênero, pois há um maior incentivo dos meninos para essa área, sobretudo com a Matemática e as Engenharias (Archer, DeWitt, \& Willis, 2014). Já as meninas não são estimuladas precocemente para estas (Sikora \& Pokropek, 2012), fazendo com que as mulheres continuem sub-representadas nas ciências exatas e tecnológicas (Nix, Roberts, \& Hughes, 2016).

Em estudo sobre as mulheres em carreiras científicas, verificou-se que as diferenças de gênero nas atitudes e expectativas sociais são evidenciadas desde o jardim de infância e aumentam a partir de então, levando o gênero feminino a menor inclinação para cursar ciência, tecnologia, engenharia e matemática na juventude, o que acaba refletindo em menor participação acadêmica delas nessas áreas científicas (Ceci, Ginther, Kahn, \& Williams, 2014). Também foi constatado em outro estudo que a atuação da mulher é maior nas ciências da Saúde (56\%) e de Linguística, Letras e Artes (50\%) e menor nas áreas de Engenharias (25\%) e de ciências Exatas e da Terra (24\%) (Fundação de Amparo à Pesquisa do Estado de São Paulo, 2017). Consequentemente, as mulheres e a sociedade acabam por perder a oportunidade de usufruir do potencial feminino inexplorado em tais áreas (Organização das Nações Unidas para Educação, Ciência e Cultura, 2017).

Ao analisar as características de personalidade dos cientistas eminentes e mais criativos (com alta produtividade), Feist (2006), Grosul e Feist (2014) constataram que eles tendem a exibir maior flexibilidade, autoconfiança, liderança, abertura à experiência, tolerância à 
frustração, motivação intrínseca, ambição e direcionamento, assim como traços ambíguos, tais como maior introversão, hostilidade e arrogância. Também Grosul e Feist (2014), ao investigar as características de profissionais criativos, ou com alta produtividade em diferentes áreas, observaram não só níveis elevados de abertura à experiência e criatividade mas também traços de psicotismo, como agressividade, obstinação e antissoabilidade. Além disso, diversos estudos que comparam homens e mulheres criativos indicam que eles têm uma personalidade andrógena, ou seja, compartilham características de personalidade mais semelhantes do que quando comparados à população geral do mesmo sexo (Runco, 2014).

As próprias condições de ensino e pesquisa são aspectos contextuais presentes na excelência científica. No caso concreto dos pesquisadores acadêmicos, as carreiras profissionais, condições materiais, laboratórios, bibliotecas e apoios financeiros de agências financiadoras podem ser decisivos (Morosini, 2009). Para tanto, várias instituições têm procurado investir em infraestrutura física e tecnológica, incentivando parcerias acadêmicas internacionais e investindo em pesquisas e publicações (Camargo, 2015), apesar do impacto que as instituições sofrem com as crises econômicas contínuas em nosso país. Por outro lado, conhecida a multiplicidade de tarefas dos pesquisadores em seus contextos acadêmicos, a conciliação de tarefas e a gestão do tempo são aspectos descritos na literatura como exercendo impacto na excelência da pesquisa, requerendo muitas vezes dos pesquisadores níveis elevados de controle emocional (Borsoi, 2012; Nunes, Hutz, Pires, \& Oliveira, 2014). Certamente o controle emocional é bastante exigido das mulheres cientistas considerando que estas necessitam balancear as atividades familiares com a carreira profissional e lutar com estereótipos sociais sobre gênero (Prado \& Fleith, 2012).

Há estudos que consideram o impacto diferencial de certas variáveis psicológicas e contextuais na excelência dos cientistas. Jindal-Snape e Snape (2006), por exemplo, verificaram que a curiosidade e a capacidade de fazer pesquisa de alta qualidade são percebidos por aqueles como fatores intrínsecos de sua motivação, assim como a necessidade subjetiva para buscar novidades, desafios e aprendizagens. De outro modo, a obtenção de premiações e distinções na área científica em que atua, o trabalho em cooperação com instituições prestigiadas internacionalmente, ser membro do conselho editorial de revistas e de periódicos, dentre outros, caracterizam o cientista talentoso de acordo com Zanotto (2006). Além disso, a ética e a integridade na atividade do cientista são fatores ressaltados pelas agências de fomento brasileiras, sendo fundamentais em todas as instâncias pertinentes ao processo de produção e divulgação de conhecimento (Santos, 2017).
Considerando que existem vários fatores de ordem cognitiva, criativa, emocional e contextual que podem contribuir para se atingir e manter um nível de excelência na produção científica, este estudo visa investigar a importância das variáveis pessoais e dos fatores contextuais na explicação da excelência em pesquisadores brasileiros. Nesse sentido, hipotetiza-se que haveria diferença predominante por gênero e tipo de área de conhecimento nos fatores não só de ordem pessoal, mas também contextual.

\section{Método}

\section{Participantes}

A amostra inicial contatada foi de 846 pesquisadores e a amostra final respondente foi de $12,88 \%$ desse total, ou seja, 109 pesquisadores, bolsistas de produtividade do CNPq 1A (33,9\%), 1B (36,6\%), 1C (23,8\%) e $1 \mathrm{D}(5,5 \%)$, sendo $50(45,8 \%)$ do sexo feminino e 59 $(54,1 \%)$ do sexo masculino. As idades oscilaram entre 36 e 81 anos $(M=58,6, D P=8,92)$, sendo a maioria (91 participantes) do estado civil de casado/união estável $(83,50 \%)$. Em termos de área científica, os participantes repartiam-se de forma bastante próxima quanto ao gênero em quatro grandes áreas, ou seja, Biológicas $(N=29$, feminino $=34 \%)$, Exatas/Tecnológicas $(N=25$, feminino $=44 \%)$, Ciências Humanas $(N=28$, feminino $=53 \%)$ e Ciências da Saúde $(N=27$, feminino $=51 \%$ ). Quanto ao tipo de instituição, a maioria dos participantes eram provenientes de instituições públicas $(84,4 \%)$.

\section{Instrumento}

Questionário do Perfil de Excelência. Instrumento elaborado pelos autores e adaptado para uma versão on-line, tendo o termo de consentimento na página inicial, que permitia o prosseguimento após ter sido aceito pelo participante. Esse questionário foi composto por quinze questões semiabertas relacionadas aos seguintes aspectos: fatores biográficos (fase da vida em que percebeu inclinação para carreira científica), influências na carreira científica (pai/mãe, professor, leituras, oportunidades, modelo profissional), aspectos cognitivos (persistência, curiosidade, flexibilidade, ausência de preconceito, imaginação), de personalidade criativa (independência de julgamento, abertura ao novo, inconformismo, liderança, busca por desafios, intuição, motivação), recursos pessoais utilizados para o sucesso no trabalho (persuasão, integridade, redes de contato) e fatores contextuais ( estímulo à produção científica, condições materiais de laboratório e bibliotecas, salários e premiações). Fazia parte do instrumento uma checklist para levantamento dos aspectos cognitivos e da personalidade dos participantes. Esse questionário foi construído 
baseado na literatura sobre os fatores determinantes da excelência profissional.

\section{Procedimentos}

Após aprovação do estudo pelo Comitê de Ética em Pesquisa com Seres Humanos sob o parecer $n^{\circ}$ 51199415.1.0000.5481, foi feita uma busca para obtenção de informações por meio da Plataforma Lattes com o seguinte critério: pesquisadores bolsistas de produtividade do CNPq 1A, 1B, 1C e 1D das áreas de Humanas, Biológicas, Saúde e Exatas/Tecnologias. Depois de identificados, os pesquisadores foram contatados via correio eletrônico, utilizando-se a plataforma Survey Monkey, com o objetivo de apresentar a proposta da pesquisa e convidá-los a participar do estudo. O tempo médio de resposta ao Questionário do Perfil de Excelência on-line foi de 6 minutos.

\section{Procedimentos de Análise de Dados}

O conteúdo das respostas foi analisado e categorizado de acordo com os referenciais teóricos e as dimensões pessoais e contextuais de análise da excelência. Após, a análise das respostas por área científica e gênero foram feitas por meio do qui-quadrado.

\section{Resultados}

$\mathrm{Na}$ Tabela 1, é descrito em que fase da vida os participantes se sentiram inclinados para uma carreira profissional de pesquisa científica. Também nesta e nas tabelas seguintes os resultados por gênero e área científica dos participantes são diferenciados conforme os objetivos do estudo.

Os resultados sugerem que a maioria dos participantes percebeu inclinação para carreira científica na fase adulta $(43,1 \%)$, embora quase $25 \%$ deles apontem essa inclinação já na sua infância. Cabe ressaltar que, enquanto a maioria dos pesquisadores do gênero feminino apresentou inclinação para carreira científica na fase adulta (54,2\%), no caso dos homens repartem-se pela infância, adolescência e idade adulta em proporções muito similares (cerca de um terço de participantes para cada faixa etária). Essa diferença nos resultados segundo o gênero apresenta-se significativa $\left[\chi^{2}(2)=6,863, p<0,05\right]$.

Tabela 1

Fase da Vida em que Houve Inclinação para Carreira Científica

\begin{tabular}{|c|c|c|c|c|c|c|c|}
\hline \multirow{2}{*}{ Fase da vida } & Geral & Feminino & Masculino & Biológicas & Exatas & Humanas & Saúde \\
\hline & $f(\%)$ & $f(\%)$ & $f(\%)$ & $f(\%)$ & $f(\%)$ & $f(\%)$ & $f(\%)$ \\
\hline Adulta & $47(43,1)$ & $27(54,2)$ & $20(33,9)$ & $5(17,2)$ & $7(28,0)$ & $16(57,1)$ & $19(70,4)$ \\
\hline Adolescência & $35(32,1)$ & $16(32,0)$ & $19(32,2)$ & $15(51,7)$ & $10(40,0)$ & $6(21,4)$ & $4(14,8)$ \\
\hline Infância & $27(24,8)$ & $7(14,0)$ & $20(33,9)$ & $9(31,0)$ & $8(32,0)$ & $6(21,4)$ & $4(14,8)$ \\
\hline
\end{tabular}

No que se refere às quatro áreas científicas, a maioria dos participantes nas áreas de Humanas e da Saúde identificam a idade adulta como fase da vida em que perceberam a sua inclinação para a carreira científica (57,1\% e 70,4\% respectivamente), enquanto nas áreas das Ciências Biológicas e Exatas tal percepção ocorreu já na fase da adolescência (51,7\% e 40\% respectivamente). Também nessas duas últimas áreas, quase um terço dos participantes associam essa sua inclinação à infância. Essa diferenciação nos resultados apresenta-se significativa $\left[\chi^{2}(6)=21,419, p<0,01\right]$.

$\mathrm{Na}$ Tabela 2, é apresentada a percepção de relevância de adultos significativos ou colegas que mais influenciaram os participantes na decisão pela carreira de pesquisa científica. No caso dos professores, não são diferenciados pelo grau de ensino (fundamental, médio e superior).

Tabela 2

Influências na Carreira Científica

\begin{tabular}{|c|c|c|c|c|c|c|c|}
\hline \multirow{2}{*}{ Pessoa } & Geral & Feminino & Masculino & Biológicas & Exatas & Humanas & Saúde \\
\hline & $f(\%)$ & $f(\%)$ & $f(\%)$ & $f(\%)$ & $f(\%)$ & $f(\%)$ & $f(\%)$ \\
\hline Pai/ Mãe & $34(28,3)$ & $16(30,7)$ & $18(26,4)$ & $13(41,9)$ & $9(30,0)$ & $9(25,7)$ & $3(12,5)$ \\
\hline Amigo(a) & $5(4,1)$ & $2(3,8)$ & $3(4,4)$ & $1(3,2)$ & $1(3,3)$ & $2(5,7)$ & $1(4,1)$ \\
\hline Professor(a) & $81(67,5)$ & $34(65,3)$ & $47(69,1)$ & $17(54,8)$ & $20(66,6)$ & $24(68,5)$ & $20(83,3)$ \\
\hline \multicolumn{8}{|l|}{ Tipo de influência } \\
\hline Incentivo/ Leituras & $57(39,3)$ & $24(34,7)$ & $33(43,4)$ & $19(50,0)$ & $17(53,1)$ & $16(34,7)$ & $8(22,8)$ \\
\hline Oportunidades & $42(28,9)$ & $24(34,7)$ & $18(23,6)$ & $12(31,5)$ & $7(21,8)$ & $13(28,2)$ & $11(31,4)$ \\
\hline Modelo profissional & $46(31,7)$ & $21(30,4)$ & $25(32,8)$ & $7(18,4)$ & $8(25,0)$ & $17(36,9)$ & $16(45,7)$ \\
\hline
\end{tabular}


Os resultados sugerem que os professores são considerados como os que mais exerceram influência na escolha da carreira científica pelos participantes de ambos os gêneros $(67,5 \%)$ e das quatro áreas das Ciências - Biológicas, Exatas, Humanas e Saúde, seguidos pelos pais $(28,3 \%)$. E, embora haja uma eventual presença da influência dos pais de acordo com o gênero e a área científica dos respondentes, verificou-se que não se observa uma diferença com significado estatístico.

Deve-se acrescentar que influência dos professores e pais se deu por meio de incentivos e leituras (39,3\%), sendo essa a via de influência mais presente sobretudo nos participantes do sexo masculino e nas três áreas científicas. Para o sexo feminino existe também uma maior referência às oportunidades $(34,7 \%)$ que aparecem na sua vida. Ao mesmo tempo se destaca a maior presença de influência de mentores como modelos profissionais nas áreas científicas de Humanas e, muito particularmente, de Saúde (45,7\%). Analisando o significado estatístico das oscilações nas frequências desses três tipos de influências segundo o gênero e a área científica dos participantes no estudo, apenas na frequência da influência como modelo de profissional se observa uma diferença com significado estatístico ao nível da área científica de atuação $\left[\chi^{2}(3)=9,483, p<0,024\right]$, traduzindo uma maior proporção de ocorrências desse tipo de influência nas áreas da Saúde e Humanas face às áreas de Exatas e Biológicas.

$\mathrm{Na}$ Tabela 3, são registradas as respostas dos participantes relativas às características de personalidade (motivação, curiosidade, intuição, liderança, busca por desafios, inconformismo/abertura ao novo, independência de julgamento e persistência) e de inteligência (flexibilidade/ausência de preconceito e imaginação) que acreditam ser importantes para a carreira científica. Ao mesmo tempo, face ao desdobramento dessa questão, são indicados também os participantes que as julgam possuir.

Tabela 3

Características de Personalidade e Intelectuais Julgadas como Importantes e Possuídas para a Carreira Científica

\begin{tabular}{|c|c|c|c|c|c|c|c|}
\hline \multirow{2}{*}{$\begin{array}{l}\text { Características que } \\
\text { julgam importantes }\end{array}$} & Geral & Feminino & Masculino & Biológicas & Exatas & Humanas & Saúde \\
\hline & $f(\%)$ & $f(\%)$ & $f(\%)$ & $f(\%)$ & $f(\%)$ & $f(\%)$ & $f(\%)$ \\
\hline Motivação & $93(13,0)$ & $43(12,5)$ & $50(13,6)$ & $25(13,8)$ & $23(14,1)$ & $22(11,0)$ & $23(13,4)$ \\
\hline Curiosidade & $92(12,9)$ & $43(12,5)$ & $49(13,3)$ & $25(13,8)$ & $23(14,1)$ & $21(10,5)$ & $23(13,4)$ \\
\hline Intuição & $43(6,0)$ & $17(4,9)$ & $26(7,0)$ & $13(7,2)$ & $14(8,5)$ & $11(5,5)$ & $9(5,2)$ \\
\hline Liderança & $50(7,0)$ & $21(6,1)$ & $29(7,9)$ & $14(7,7)$ & $4(2,4)$ & $17(8,5)$ & $15(8,7)$ \\
\hline Busca por desafios & $59(8,3)$ & $30(8,7)$ & $29(7,9)$ & $15(8,3)$ & $16(9,8)$ & $13(6,5)$ & $14(8,1)$ \\
\hline Inconformismo/abertura ao novo & $86(12,1)$ & $47(13,7)$ & $39(10,6)$ & $18(10,0)$ & $16(9,8)$ & $34(17,0)$ & $18(10,5)$ \\
\hline Independência de julgamento & $47(6,6)$ & $26(7,5)$ & $21(5,7)$ & $9(5,0)$ & $11(6,7)$ & $13(6,5)$ & $14(8,1)$ \\
\hline Persistência & $87(12,2)$ & $46(13,4)$ & $41(11,1)$ & $24(13,3)$ & $22(13,4)$ & $21(10,5)$ & $20(11,6)$ \\
\hline Flexibilidade/ausência de preconceito & $96(13,5)$ & $48(13,9)$ & $48(13,0)$ & $22(12,2)$ & $19(11,6)$ & $32(16,0)$ & $23(13,4)$ \\
\hline Imaginação & $57(8,0)$ & $22(6,4)$ & $35(9,5)$ & $15(8,3)$ & $15(9,2)$ & $15(7,5)$ & $12(7,0)$ \\
\hline \multicolumn{8}{|l|}{ Características que julgam possuir } \\
\hline Motivação & $88(12,8)$ & $37(12,2)$ & $51(13,3)$ & $27(13,8)$ & $23(14,5)$ & $17(9,9)$ & $21(12,9)$ \\
\hline Curiosidade & $80(11,6)$ & $34(11,2)$ & $46(12,0)$ & $25(12,8)$ & $17(10,7)$ & $19(11,1)$ & $19(11,7)$ \\
\hline Intuição & $42(6,1)$ & $14(4,6)$ & $28(7,3)$ & $10(5,1)$ & $13(8,2)$ & $9(5,2)$ & $10(6,1)$ \\
\hline Liderança & $55(8,0)$ & $24(7,9)$ & $31(8,0)$ & $19(9,7)$ & $6(3,7)$ & $14(8,1)$ & $16(9,8)$ \\
\hline Busca por desafios & $64(9,3)$ & $30(9,9)$ & $34(8,8)$ & $17(8,7)$ & $15(9,4)$ & $15(8,7)$ & $17(10,4)$ \\
\hline Inconformismo/abertura ao novo & $75(10,9)$ & $37(12,2)$ & $38(9,9)$ & $18(9,2)$ & $14(8,8)$ & $28(16,3)$ & $15(9,2)$ \\
\hline Independência de julgamento & $49(7,1)$ & $22(7,2)$ & $27(7,0)$ & $11(5,6)$ & $13(8,2)$ & $14(8,1)$ & $11(6,7)$ \\
\hline Persistência & $84(12,2)$ & $43(14,1)$ & $41(10,7)$ & $23(11,7)$ & $22(13,9)$ & $19(11,1)$ & $20(12,3)$ \\
\hline Flexibilidade/ausência de preconceito & $104(15,1)$ & $46(15,1)$ & $58(15,1)$ & $30(15,3)$ & $25(15,8)$ & $25(14,6)$ & $24(14,8)$ \\
\hline Imaginação & $45(6,5)$ & $16(5,2)$ & $29(7,5)$ & $15(7,6)$ & $10(6,3)$ & $11(6,4)$ & $9(5,5)$ \\
\hline
\end{tabular}

Numa primeira análise dos resultados na Tabela 3, observa-se uma dispersão das respostas dos participantes do estudo quanto às características de personalidade e intelectuais que consideram importantes para a carreira científica. Entre os gêneros, poucas diferenças são notadas, embora haja diferença significativa entre eles para a característica persistência $\left[\chi^{2}(1)=8,511, p<0,004\right]$ e para inconformismo/abertura ao novo $\left[\chi^{2}(1)=4,825\right.$, $p<0,028]$, mais valorizadas pelas mulheres, enquanto os homens valorizam mais a intuição, embora essa diferença não seja significativa. 
Quanto às áreas científicas onde exercem a sua carreira de pesquisa, como pode ser visto na Tabela 3, alguns contrastes parecem existir entre os participantes de Exatas e de Humanas. Comparando esses dois grupos em particular, os de Exatas parecem valorizar mais, as características de motivação, curiosidade, intuição e busca de desafios. Por sua vez, os profissionais da área das Humanas estão em maior percentagem na valorização da liderança, estando essa característica em menor presença entre os participantes de Exatas $\left[\chi^{2}(3)=12,556\right.$, $p<0,006]$, assim como a de inconformismo/abertura ao novo $\left[\chi^{2}(3)=12,089, p<0,007\right]$ e flexibilidade (na importância atribuída).

No que se refere às características de personalidade e intelectuais que os participantes julgam possuir, também se nota uma dispersão nas respostas. As mulheres se sentem mais inconformistas e mais persistentes nas suas carreiras, enquanto os homens se percebem em maior proporção como possuindo intuição $\left[\chi^{2}(1)=4,326, p<0,038\right]$. Devese acrescentar que os participantes de Biológicas e Saúde se auto avaliam em maior percentagem como possuindo a característica de liderança nas suas carreiras, sendo essa oscilação estatisticamente significativa $\left[\chi^{2}(3)=10,471\right.$, $p<0,015]$. Por sua vez, os participantes de Humanas são os que menos se auto avaliam em termos de motivação, comparando com outras áreas, sendo essa diferença significativa $\left[\chi^{2}(3)=12,259, p<0,007\right]$.

$\mathrm{Na}$ Tabela 4, são apresentados os recursos pessoais que os participantes utilizam para que obtenham sucesso na sua atividade profissional.

Tabela 4

Recursos Pessoais Utilizados para o Sucesso no Trabalho

\begin{tabular}{lccccccc}
\hline \multirow{2}{*}{ Fatores contextuais } & Global & Feminino & Masculino & Biológicas & Exatas & Humanas & Saúde \\
\cline { 2 - 7 } & $f(\%)$ & $f(\%)$ & $f(\%)$ & $f(\%)$ & $f(\%)$ & $f(\%)$ & $f(\%)$ \\
\hline Persuasão/persistência & $92(37,5)$ & $44(38,9)$ & $48(36,3)$ & $22(34,9)$ & $24(44,4)$ & $24(37,5)$ & $22(34,3)$ \\
Postura íntegra & $91(37,1)$ & $45(39,8)$ & $46(34,8)$ & $26(41,2)$ & $19(35,1)$ & $21(32,8)$ & $25(39,0)$ \\
Rede de contatos & $62(25,3)$ & $24(21,2)$ & $38(28,7)$ & $15(23,8)$ & $11(20,3)$ & $19(29,6)$ & $17(26,5)$ \\
\hline
\end{tabular}

Os recursos citados como mais utilizados para o alcance do sucesso no trabalho foram a persuasão/persistência e a postura íntegra na sua atividade profissional, ambas relativamente próximas na amostra geral e por gênero, havendo uma oscilação nas percentagens de resposta quando as áreas científicas são consideradas. Já a utilização de rede de contatos é a estratégia percentualmente menos presente nas respostas da amostra $(25,3 \%)$. Por exemplo, a persuasão/persistência está mais presente na área das Exatas (44,4\%), estando menos presente nesse subgrupo de pesquisadores o uso de redes de contatos $(20,3 \%)$. Por sua vez, na área de
Biológicas, e em parte na Saúde, a postura íntegra supera a percentagem de respostas alusivas à persuasão/persistência. Mesmo assim, tomando as oscilações de cada um dos recursos ou estratégia implementada pelos participantes para aumentarem o sucesso na sua atividade profissional, em nenhum caso se observou significância estatística ao considerar o gênero e a área científica dos participantes.

Na Tabela 5, são apresentados os resultados das respostas dos participantes à questão sobre as condições ou fatores favoráveis à qualidade da sua pesquisa existentes nas instituições de ensino superior.

Tabela 5

Fatores Contextuais do Ensino Superior que Influenciam a Excelência

\begin{tabular}{lccccccc}
\hline \multicolumn{1}{c}{$\begin{array}{c}\text { Fatores institucionais } \\
\text { do ensino superior }\end{array}$} & Global & Feminino & Masculino & Biológicas & Exatas & Humanas & Saúde \\
\cline { 2 - 7 } & $f(\%)$ & $f(\%)$ & $f(\%)$ & $f(\%)$ & $f(\%)$ & $f(\%)$ & $f(\%)$ \\
\hline Estímulo à produção científica & $91(25,2)$ & $44(25,1)$ & $47(25,4)$ & $25(27,1)$ & $22(26,1)$ & $23(24,2)$ & $21(23,5)$ \\
Condições materiais/ & $118(32,7)$ & $57(32,5)$ & $61(32,9)$ & $29(31,5)$ & $24(28,5)$ & $34(35,7)$ & $31(34,8)$ \\
laboratórios/bibliotecas & & & & & & & \\
Financiamento & $86(23,8)$ & $41(23,4)$ & $45(24,3)$ & $22(23,9)$ & $22(26,1)$ & $21(22,1)$ & $21(23,5)$ \\
Salário/premiações & $65(18,0)$ & $33(18,8)$ & $32(17,2)$ & $16(17,3)$ & $16(19,0)$ & $17(17,8)$ & $16(17,9)$ \\
\hline
\end{tabular}

Os resultados sugerem que os recursos (condições materiais, laboratórios e bibliotecas) disponíveis nas instituições de ensino superior são fatores mais frequentes nas respostas dos participantes $(32,7 \%)$, estando o 
reconhecimento da importância desses recursos também em primeiro lugar, analisando a amostra tanto quanto o gênero quanto por área científica. O estímulo à produção científica e o financiamento dos projetos surgem muito próximos nas respostas dos participantes, sendo valorizados por cerca de $25 \%$ dos respondentes. Por último, cerca de $18 \%$ da amostra total dos participantes valorizam mais a qualidade da sua produção do que os salários e as premiações auferidas. Analisando a valorização apontada para essas várias fontes de estímulo por parte dos participantes segundo o gênero e a área científica de atuação, em nenhum caso foram observadas diferenças estatisticamente significativas.

\section{Discussão}

Este estudo objetivou investigar as variáveis pessoais e contextuais que afetam pesquisadores que atingem excelência. Os resultados indicaram que o gênero e a área de conhecimento afetam mais as características pessoais dos pesquisadores, do que os fatores contextuais, para os quais não foram encontradas diferenças significativas. Houve diferença significativa entre os gêneros no período em que perceberam inclinação para carreira científica, apresentando as mulheres tal predisposição mais na fase adulta enquanto a maioria dos homens demonstrou esse interesse já na infância e adolescência, o que parece mais de acordo com os autores que referem essa inclinação ocorre já na adolescência (Tai et al., 2006). Estes resultados podem decorrer da forma como a escola e os seus professores identificaram os talentos nos seus alunos e a atenção que lhes deram, podendo os meninos terem recebido mais estímulo pelas atividades científicas do que as meninas, como apontam algumas pesquisas nessa área (Buchmann et al., 2008; Wu, 2005).

A idade do reconhecimento do interesse pela profissão diferiu também pelas quatro áreas científicas. A maior parte dos pesquisadores das áreas das Humanas e da Saúde mencionou a idade adulta como fase da vida em que perceberam a sua inclinação para a carreira científica, ocorrendo esse reconhecimento na adolescência nas áreas das Ciências Biológicas e Exatas. Devese acrescentar que, nessas duas últimas áreas, quase um terço dos participantes associam essa sua inclinação à infância, podendo sugerir uma identificação mais precoce de talentos nessas áreas curriculares. Para Archer, DeWitt e Willis (2014), as ciências, matemática e engenharias são percebidas como atividades para meninos, o que pode explicar o resultado nas duas últimas áreas do conhecimento citadas que são mais ocupadas pelo sexo masculino. Por outro lado, as meninas não são estimuladas precocemente para a matemática, o que pode influenciar no fato das mulheres continuarem a estar sub-representadas nos campos das ciências exatas e tecnológicas (Fundação de Amparo à Pesquisa do Estado de São Paulo, 2017; Nix et al., 2016).
No que se refere à mentoria, os professores são os mais citados pelos participantes de ambos os gêneros e nas quatro áreas científicas como tendo influenciado as suas carreiras. Esse dado vai de encontro à investigação que aponta a qualidade do professor como determinante no processo de identificação e desenvolvimento de um estudante talentoso (Osborne et al., 2004; Pruitt \& Wallace, 2012; Bakir \& Öztekin, 2014), desde a educação primária (Baron \& Chen, 2012) até o ensino superior (Russell, Hancock, \& McCullough, 2007). Para o sexo feminino existe uma referência relevante às oportunidades parecendo haver maior dependência de variáveis de contexto numa sociedade cuja igualdade de gênero ainda não foi conseguida.

Os pesquisadores também relataram a influência de mentores como modelos profissionais, tendo isso ocorrido mais para as áreas de Saúde e Humanas do que para as áreas de Exatas e Biológicas. Conforme Salehjee e Watts (2015), o modelo do professor e a identificação para com determinada disciplina vista na escola são percebidos como fatores decisivos na escolha de meninos e meninas pela atividade científica. Para Fleming, Burnham e Huskins (2012) e Gonzalez (2001), a mentoria é um processo importante ao apoiar e estimular o aluno a ir além das aprendizagens curricularmente exigidas numa postura de progressiva reflexão e construção de conhecimentos científicos.

Dentre as caraterísticas pessoais mais apreciadas e possuídas pelos participantes, notou-se diferença significativa entre os gêneros para as características persistência, intuição, inconformismo e abertura ao novo. As mulheres se percebem como mais persistentes nas suas carreiras e valorizam mais o inconformismo e abertura ao novo, enquanto os homens valorizam mais a intuição. Deve ser ressaltado, entretanto, que tais diferenças de gênero não são confirmadas em outros estudos com homens e mulheres eminentes, pois essas pessoas possuem semelhanças nas características de personalidade quando comparados à população do mesmo sexo, ou seja, apresentam uma personalidade andrógena (Runco, 2014). Além disso, as características destacadas pelos participantes referem-se às várias fortalezas dos campos da personalidade e da motivação com que a Psicologia Positiva descreve o bem-estar, a realização pessoal e o sucesso profissional (Seligman \& Csikszentmihalyi, 2000), podendo assim compreender melhor o seu impacto sobre a excelência científica.

Considerando as áreas científicas onde os participantes exercem a sua carreira de pesquisa, observou-se diferença significativa dentre aqueles das Humanas, Exatas, Biológicas e da Saúde nas características de liderança, inconformismo e abertura ao novo. Os pesquisadores de Humanas valorizam mais estas características, além de se auto avaliarem como mais inconformistas e abertos à novidade, apesar de se sentirem menos motivados. Já os participantes das áreas da Saúde e Biológicas 
apontam características de liderança como específicas da sua área. Essas caraterísticas tendem a estar também presentes em estudos similares com cientistas eminentes de diferentes áreas do conhecimento, que exibem maior flexibilidade, motivação intrínseca, maior abertura à experiência e maior tolerância à frustração e liderança, mas também tendências ao psicotismo, tais como hostilidade, introversão, agressividade, embora estas últimas não tenham sido observadas neste estudo (Feist, 2006; Grosul \& Feist, 2014).

Com relação aos recursos que os participantes mobilizam para o êxito do seu trabalho, não foram verificadas diferenças significativas considerando o gênero e a área científica deles. Entretanto, as estratégias mais citadas pelos pesquisadores, de uma maneira geral, foram a persuasão/persistência e postura íntegra em suas atividades profissionais. Por sua vez, na área de Biológicas, e em parte na Saúde, a postura íntegra superou a percentagem de respostas alusivas à persuasão/persistência, sugerindo a relevância das questões humanas e éticas nos seus domínios de pesquisa. A ética e a integridade na atividade do cientista são fatores ressaltados pelas agências de fomento brasileiras, sendo fundamentais em todas as instâncias pertinentes ao processo de produção e divulgação de conhecimento (Santos, 2017). A utilização de rede de contatos foi a estratégia percentualmente menos presente nas respostas da amostra, sugerindo a presença, sobretudo de caraterísticas baseadas na autonomia, responsabilidade, auto eficácia e competência pessoal.

Quanto aos fatores contextuais percebidos como influenciáveis no seu trabalho, observou-se que os recursos relacionados às condições materiais, laboratórios e bibliotecas disponíveis foram os mais frequentemente citados nas respostas dos participantes em geral, podendo ser decisivos para o desempenho dos cientistas (Morosini, 2009). Por sua vez, os salários e as premiações auferidas foram os menos destacados pelos pesquisadores homens e mulheres das quatro áreas de conhecimento, podendo-se inferir que o impacto e a qualidade de suas produções são percebidos como mais importantes do que o reconhecimento social e as distinções recebidas, vistas como consequentes dos resultados de seus trabalhos (Zanotto, 2006). A motivação intrínseca parece ser decisiva aos cientistas eminentes, por exemplo, retratada pela dedicação, abertura às novidades, e gosto por desafios (JindalSnape \& Snape, 2006).

Conclui-se, portanto, que os fatores pessoais são influenciados pelo gênero e área de conhecimento para o sucesso profissional do cientista. Tais características, por sua vez, têm grande influência na fase da vida em que há inclinação pela carreira, na escolha do mentor e da área científica. Assim sendo, fatores de ordem pessoal auxiliam mais na compreensão da excelência científica do que os de ordem contextual. Sem dúvida, as questões de gênero são extremamente relevantes para a escolha e ascensão na carreira científica. Quanto aos fatores contextuais, embora possam contribuir para a produção científica, estes não são vistos como sendo os mais importantes na excelência científica.

Algumas limitações deste estudo devem ser mencionadas. Por um lado, o tamanho da amostra foi reduzido face ao número de cientistas que participaram, ao mesmo tempo o foco da amostra foi nas áreas das ciências Humanas, Biológicas, Saúde e Exatas, não sendo possível conhecer cientistas de outras áreas de conhecimento. No futuro será necessário comparar dados de auto relato com avaliações por pares da mesma área científica. Por outro lado, fará sentido investigar a excelência em outros domínios, como as artes, literatura e esportes.

\section{Referências}

Almeida, L. S., \& Wechsler, S. M. (2015). Excelência profissional: A convergência necessária de variáveis psicológicas. Estudos de Psicologia, 32(4), 767-775. doi:10.1590/0103-166X2015000400019

Araújo, L. S., Cruz, J. F. A., \& Almeida, L. S. (2007). Excelência humana: Teorias explicativas e papel determinante do professor. Psicologia, Educação e Cultura, XI(2), 197-221.

Archer, L., DeWitt, J., \& Willis, B. (2014). Adolescent boys' science aspirations: Masculinity, capital, and power. Journal of Research in Science Teaching, 51(1), 1-30. doi:10.1002/tea.21122

Bakir, S., \& Öztekin, E. (2014). Creative thinking levels of preservice science teachers in terms of different variables. Journal of Baltic Science Education, 13(2). 231-242. Recuperado de http://www.scientiasocialis.lt/jbse/files/pdf/vol13/231-242.Bakir_JBSE_Vol.13_No.2.pdf

Baron, A., \& Chen, H. L. S. (2012). Looking in a science classroom: Exploring possibilities of creative cultural divergence in science teaching and learning. Cultural Studies of Science Education, 7(1), 93-101. doi: 10.1007/s11422-012-9402-6

Borsoi, I. C. F. (2012). Trabalho e produtivismo: Saúde e modo de vida de docentes de instituições públicas de ensino superior. Cadernos de Psicologia Social do Trabalho, 15(1), 81-100. doi: 10.11606/issn.1981-0490.v15i1p81-100

Buchmann, C., DiPrete, T. A., \& McDaniel, A. (2008). Gender inequalities in education. Annual Review of Sociology, $34,319-337$. doi:org/10.1146/annurev.soc.34.040507.134719

Camargo, M. S. (2015). Indicadores da educação superior brasileira de 2003 a 2013: Dados e resultados das políticas públicas implementadas. Atos de Pesquisa em Educação, 1(10), 176-202. doi: 10.7867/1809-0354.2015v1n10p176-202

Ceci, S. J., Ginther, D. K., Kahn, S., \& Williams, W. M. (2014). Women in academic science: A changing landscape. Psychological Science in the Public Interest, 15(3), 75-141. doi: 10.1177/1529100614541236 
Dabney, K. P., Chakraverty, D., \& Tai, R. H. (2013). The association of family influence and initial interest in science. Science Education, 97(3), 395-409. doi:org/10.1002/sce.21060

Dou, R., Hazari, Z., Dabney, K., Sonnert, G., \& Sadler, P. (2019). Early informal STEM experiences and STEM identity: The importance of talking science. Science Education, 103(3), 623-637. doi: 10.1002/sce.21499

Fundação de Amparo à Pesquisa do Estado de São Paulo (2017). Mulheres na ciência. Recuperado de https://revistapesquisa.fapesp. br/2017/09/22/mulheres-na-ciencia-8/

Feist, G. J. (2006). How development and personality influence scientific thought, interest, and achievement. Review of General Psychology, 10(2), 163-182. doi:10.1037/1089-2680.10.2.163

Fleming, M., Burnham, E. L., \& Huskins, W. C. (2012). Mentoring translational science investigators. JAMA, 308(19), $1981-1982$. doi:10.1001/jama.2012.14367

Garcia-Santos, S. C., Almeida, L. S., \& Werlang, B. S. G. (2012). Excelência Humana: A contribuição da personalidade. Paidéia, 22(5), 251259. doi:10.1590/S0103-863X2012000200011

Gonzalez, C. (2001). Undergraduate research, graduate mentoring, and the university's mission. Science, 293(5535), 1624-1626. doi: $10.1126 /$ science. 1062714

Gould, J. C., \& Weeks, V. (2005). Science starts early. Science Education for Gifted Students, 3, 38-42. doi:10.1126/science.1195221

Grosul, M., \& Feist, G. J. (2014). The creative person in science. Psychology of Aesthetics, Creativity, and the Arts, 8(1), 30-43. doi:10.1037/ a0034828

Hutz, C. S., \& Pacico, J. C., (2016). Psicologia Positiva: Avanços de uma nova abordagem. Em Corrêa, A. P. (Ed.). Psicologia Positiva: Teoria e Prática (78-84). São Paulo: Leader

Jindal-Snape, D., \& Snape, J. B. (2006). Motivation of scientists in a government research institute: Scientists' perceptions and the role of management. Management Decision, 44(10), 1325-1343. doi:10.1108/00251740610715678

Jones, G., Taylor, A., \& Forrester, J. H. (2011). Developing a scientist: A retrospective look. International Journal of Science Education, 33(12), 1653-1673. doi:10.1080/09500693.2010.523484

Monteiro, S., Almeida, \& L. S., Vasconcelos, R. (2009). O papel docente na formação de alunos de excelência na engenharia: Dados de um estudo qualitativo na Universidade do Minho. World Congress on Communication and Arts. Abril 19 a 22, São Caetano do Sul, BRAZIL.

Morosini, M. C. (2009). A pós-graduação no Brasil: Formação e desafios. Revista Argentina de Educación Superior, 1(1), 125-152. Recuperado de https://dialnet.unirioja.es/servlet/articulo?codigo $=6485544$

Nix, S., Roberts, K. L., \& Hughes, R. (2016). Female Undergraduate STEM Persistence: A focus on the role of living and learning communities. Journal of Women and Minorities in Science and Engineering, 22(4), 1-33. doi: 10.1615/JWomenMinorScienEng.2016016349

Nunes, M. F. O., Hutz, C. S., Pires, J. G., \& Oliveira, C. M. (2014). Subjective well-being and time use of Brazilian PhD professors. Paidéia (Ribeirão Preto), 24(59), 379-387. doi:10.1590/1982-43272459201412

Olszewski-Kubilius, P., \& Thomson, D. (2015). Talent development: What does it look like in practice? Gifted Child Today, 38, 5-6. doi:10.1177/1076217514556280

Osborne, J., Erduran, S., \& Simon, S. (2004). Enhancing the quality of argumentation in school science. Journal of research in science teaching, 41(10), 994-1020. doi:org/10.1002/tea.20035

Prado, R. M., \& Fleith, D. S. (2012). Pesquisadoras brasileiras: Conciliando talento, ciência e família.Arquivos Brasileiros de Psicologia, 64(2),1934. Recuperado de https://www.redalyc.org/pdf/2290/229023851003.pdf

Pruitt, S. L., \& Wallace, C. S. (2012). The effect of a state department of education teacher mentor initiative on science. Achievement. Journal of Science Teacher Education, 23(4), 367-385. doi:10.1007/s 10972-0 12-9280-5

Renzulli, J. S. (2005). The three-ring conception of giftedness: A developmental model for promoting creative productivity. Em R. J. Sternberg \& J. Davidson (Eds.), Conceptions of giftedness (pp. 246-279). New York, NY: Cambridge University Press.

Renzulli, J. S., Siegle, D., Reis, S. M., Gavin, M. K., \& Reed, R E. S. (2009). An investigation of the reliability and factor structure of four new Scales for Rating the Behavioral Characteristics of Superior Students. Journal of Advanced Academics 22(1), 84-108. doi:10.1177/1932202X0902100105

Runco, M. A. (2014). Creativity - Theories and themes: Research, development and practice. San Diego, California: Elsevier.

Russell, S. H., Hancock, M. P., \& McCullough, J. (2007). Benefits of undergraduate research experiences. Science, 316(5824), 548-549. doi:10.1126/science.1140384

Salehjee, S., \& Watts, M. (2015). Science lives: School choices and 'natural tendencies'. International Journal of Science Education, 37(4), 727743. doi: 10.1080/09500693.2015.1013075

Santos, L. H. L. D. (2017). Sobre a integridade ética da pesquisa. Ciência e Cultura, 69(3), 4-5.

Seligman, M., Csikszentmihalyi, M. (2000). Positive Psychology: An introduction. American Psychologist, 55(1), 5-14. doi:10.1037/0003066X.55.1.5

Shavinina, L. V. (2009). Scientific talent: The case of Nobel Laureates. Em L. V. Shavinina (Ed.), International handbook on giftedness (pp. 649-669). New York, NY: Springer.

Sikora, J., \& Pokropek, A. (2012). Gender segregation of adolescent science career plans in 50 countries. Science Education, 96(2), $234-264$. doi:10.1002/sce.20479

Tai, R. H., Qi Liu, C., Maltese, A. V., \& Fan, X. (2006). Planning early for careers in science. Science, 312, 1143-1145. doi:10.1126/ science. 1128690

Trost, G. (2000). Prediction of excellence in school, higher education, and work. Em K. Heller, F. Mönks, R. Sternberg \& R. Subotnik (Eds.), International Handbook of Giftedness and Talent (pp. 317-327). Oxford, UK: Elsevier.

Organização das Nações Unidas para Educação, Ciência e Cultura (2017). Mulheres e meninas na ciência. Recuperado de http://www.unesco. org/new/pt/brasilia/natural-sciences/science-and-technology/women-and-girls-in-science/ 
Wu, E. H. (2005). Factors that contribute to talented performance: A theoretical model from a Chinese perspective. Gifted Child Quarterly, $49(3), 231-246$.

Zanotto, E. D. (2006). The scientists pyramid. Scientometrics, 69(1), 175-181. doi: 10.1007/s11192-006-0134-9

\section{Sobre os autores}

Maria Célia Bruno Mundim é pós-doutora pelo Programa de Pós-Graduação em Psicologia da Pontifícia Universidade Católica de Campinas.

Solange Muglia Wechsler é professora doutora do Programa de Pós-Graduação em Psicologia da Pontifícia Universidade Católica de Campinas.

Leandro da Silva Almeida é professor catedrático do Instituto de Educação/Universidade do Minho - Portugal. 